\title{
Fuel Optimal Maneuvers for Multispacecraft Interferometric Imaging Systems
}

\author{
Suman Chakravorty* and Jaime Ramirez ${ }^{\dagger}$ \\ Texas A\&M University, College Station, Texas 77843-3141
}

DOI: $10.2514 / 1.20178$

\begin{abstract}
In this paper, the design of minimum-fuel maneuvers for multispacecraft interferometric imaging systems is studied. It is argued that the underlying optimization problem is computationally intractable, through its similarity to the traveling salesman problem, and through an optimal control argument, and thus it is necessary to resort to heuristics in order to solve the problem. The design of minimum-fuel spiral maneuvers is considered in defining the constraints on the coverage of the $u-v$ plane. It is shown that the geometric design problem, the optimization problem obtained by fixing the angular rate of the spiral, and the kinematic design problem, obtained by fixing the spiraling rate of the spiral, are both convex in deep space, that is, perturbation free motion, and in near-Earth orbits. As an application of the methodology developed, fuel optimal maneuvers are found for a deep space imaging application and the fuel consumption and power requirements of the system are calculated to gain knowledge about the feasibility of such maneuvers.
\end{abstract}

\begin{tabular}{|c|c|c|}
\hline & & Nomenclature \\
\hline$\hat{A}_{p}$ & $=$ & Fourier transform of the field of view \\
\hline$a_{r}$ & $=$ & radial acceleration \\
\hline$a_{t}$ & $=$ & tangential acceleration \\
\hline$C$ & $=$ & $\begin{array}{l}\text { parameter indicating the factor of } \\
\text { misclassification of the image feature }\end{array}$ \\
\hline$E|x|$ & $=$ & expected value of $x$ \\
\hline$e$ & $=$ & image error \\
\hline$\hat{I}$ & $=$ & actual image \\
\hline$\hat{I}_{p}$ & $=$ & formed image \\
\hline$I_{\mathrm{sp}}^{p}$ & $=$ & specific impulse \\
\hline$k$ & $=$ & expansion rate of the spiral maneuver \\
\hline$M$ & $=$ & modulation transfer function \\
\hline$m$ & $=$ & number of rings given by the configuration \\
\hline$N$ & $=$ & noise in the image process \\
\hline$n_{s}$ & $=$ & number of spacecraft in the maneuver \\
\hline$q$ & $=$ & $\begin{array}{l}\text { variable in the optimization problem describing } \\
\text { the time spent at a given frequency }\end{array}$ \\
\hline$R$ & $=$ & radius of space plane coverage \\
\hline$r$ & $=$ & radial distance to the center of maneuver \\
\hline $\sup _{x \in S} f(x)$ & $=$ & supremum value of $f(x)$ for $x \in S$ \\
\hline$T$ & $=$ & time of maneuver \\
\hline$v_{r}$ & $=$ & radial velocity \\
\hline$v_{t}$ & $=$ & tangential velocity \\
\hline $\bar{z}$ & $=$ & distance to the target \\
\hline$\alpha$ & $=$ & $\begin{array}{l}\text { proportionality factor of distance between } \\
\text { spacecraft }\end{array}$ \\
\hline$\beta$ & $=$ & $\begin{array}{l}\text { multiplicity factor given the symmetry of the } \\
\text { formation }\end{array}$ \\
\hline $\bar{\Delta}$ & $=$ & $\begin{array}{l}\text { parameter proportional to the required light } \\
\text { acquisition }\end{array}$ \\
\hline$\Delta r_{m n}$ & $=$ & distance between the $m$ th and the $n$th spacecraft \\
\hline & $=$ & wavelength of radiation \\
\hline
\end{tabular}

Presented as Paper 159 at the 15th AAS/AIAA Space Flight Mechanics Meeting 2005, Copper Mountain, CO, 23-27 January 2005; received 21 September 2005; revision received 13 March 2006; accepted for publication 13 March 2006. Copyright ( 92006 by Suman Chakravorty. Published by the American Institute of Aeronautics and Astronautics, Inc., with permission. Copies of this paper may be made for personal or internal use, on condition that the copier pay the $\$ 10.00$ per-copy fee to the Copyright Clearance Center, Inc., 222 Rosewood Drive, Danvers, MA 01923; include the code $\$ 10.00$ in correspondence with the CCC.

*Assistant Professor, Department of Aerospace Engineering, 741D H.R. Bright Building, 3141 TAMU; schakrav@tamu.edu.

${ }^{\dagger}$ Graduate Assistant, Department of Aerospace Engineering.

$\begin{array}{lll}\mu & = & \text { rate of arrival of photons to one collecting } \\ & \text { telescope } \\ v & = & \text { frequency } \\ \rho & = & \text { ratio between radius of coverage and size of disk } \\ & \text { in the frequency plane }\end{array}$

\section{Introduction}

$\mathbf{T}$ HE research presented in this paper is motivated by the prospect of taking high resolution images of extrasolar planets at distances of up to 15 parsecs ${ }^{\ddagger}$ and other high resolution imaging applications. This high resolution imaging is to be performed by a multispacecraft interferometric imaging system (MSIIS). Different technologies that could be used for these missions have been studied $[1,2]$.

It is known that if the goal of imaging is formulated as the correct classification of the formed images, satisfactory imaging by an MSIIS is analogous to the "painting" of a large resolution disk with smaller "coverage" disks or "paintbrushes" while maintaining a minimum thickness of paint [3-7]. In this paper the design of minimum-fuel maneuvers subject to the above mentioned constraints is considered. It is shown that the minimum-fuel problem is similar to the traveling salesman problem [8,9], which is known to be computationally intractable when the number of cities is large. In such cases, heuristics need to be used in order to make the problem tractable. Spirals form a class of trajectories that satisfy the coverage constraints and it was shown in previous work that the space of spiral maneuvers forms the dominant set for minimum time problems [4-6]. In this paper, minimum-fuel spiral maneuvers are considered. It is shown that the geometric design problem, obtained by fixing the angular rate in the minimum-fuel problem, and the kinematic design problem, obtained by fixing the spiraling rate in the minimum-fuel problem, are convex and therefore can be solved conveniently for very large dimensions. The minimum-fuel problem is numerically solved in deep space (i.e., perturbation free or gravity free motion) for arbitrary number of spacecraft and the constraints that such maneuvers place on the development of appropriate propulsion technologies.

The problem of design of MSIIS is related to the fields of synthetic aperture optics and formation flying. The relationship of the present work to these topics is discussed next. The topic of long baseline interferometry falls under the category of synthetic aperture optics

${ }^{\ddagger}$ NASA Origins: Missions. Planet Imager, http://origins.jpl.nasa.gov/ missions/missions.html\#pi [cited 11 July 2006] 
[10], that was first developed in the context of synthetic aperture radars (SAR) [11]. The method consists of emulating a large optical instrument by a number of smaller ones and combining their contributions in a proper way to obtain an image that has resolution comparable to that of the large optical instrument. For a discussion of the various metrics used in the optimization of these systems, please refer to previous work [4]. All the above mentioned designs optimize the locations of the constituent telescopes such that some metric of image quality is maximized. Thus, these correspond to static optimization problems. However, for an MSIIS, due to the high resolution requirements, the "design variables" are the trajectories of the constituent spacecraft. The explicit dependence of the (MTF) on the trajectories of the constituent spacecraft has been defined in previous work [4,7]. Also, it is shown that the design of an MSIIS reduces to a trajectory optimization problem, where some resource utilization of the system is minimized while satisfying the imaging constraints placed on the trajectories of the constituent spacecraft. Some authors have proposed various different maneuvers and schemes for the imaging problem and have compared the different approaches [12-15]. However, an optimization problem for the design of such systems, for more than three spacecraft, has not been posed or solved. In previous work, a minimum time problem is solved under certain assumptions [4-6]. In this paper, the minimumfuel problem is formulated for arbitrary number of spacecraft, and its computational intractability is shown. Therefore, an heuristic approach is used to find a solution of the problem in the space of spiral maneuvers.

The original contributions of this paper are as follows:

1) The computational intractability of the minimum-fuel problem for the design of maneuvers for an MSIIS is shown, through its similarity to the traveling salesman problem and an optimal control argument, and therefore, the need to resort to heuristics in order to solve the problem. The heuristic proposed is the class of spiral trajectories because they satisfy the coverage constraints in a very straightforward and intuitive fashion.

2) The problem is posed with respect to the space of spirals and the minimum-fuel double pantograph problem obtained. It is shown that the kinematic design problem and the geometric design problems, obtained by fixing the spiral rate and the angular rate, respectively, in the minimum-fuel problem, are convex in free space and for nearEarth operation, and thus amenable to solution even for very large dimensions.

3) The fuel optimal spiral maneuvers are obtained and the expected behavior of solutions corroborated, namely that minimumfuel consumption is achieved by the loosest spiral maneuver, that is, the spiral with the largest spiraling rate. Further, the fuel expenditure of such maneuvers is evaluated and it is concluded that such imaging maneuvers might be feasible using current/proposed propulsion technologies.

The rest of the paper is organized as follows. In Sec. II, the problem of design of an MSIIS is formulated. In Sec. III, the imaging problem is framed as a painting problem and its intractability shown. In Sec. IV, the minimum-fuel problem with respect to spiral trajectories is posed and the minimum-fuel double pantograph problem obtained. In Sec. V, it is shown that the kinematic design and geometric design problem are convex in deep space and for nearEarth operation. In Sec. VI, numerical solutions are obtained to the fuel optimal control problem for spiral maneuvers in deep space, with multiple spacecraft and the implications of the results discussed, with regard to the feasibility of such maneuvers.

\section{Modeling and Problem Formulation}

In previous work [4,7], it was shown that the imaging constraints on an MSIIS translate into trajectory constraints on the constituent spacecraft of the system. In this section, the problem of designing minimum-fuel maneuvers for an MSIIS is posed such that the imaging constraints are satisfied. Some results from that earlier work are here recounted $[3,4,7]$.

The formation of the image in an MSIIS can be represented as follows in the spatial frequency (wave number) domain:

$$
\hat{I}_{p}(v)=\hat{I}(v)+e(v)
$$

Also, it is known that

$$
e(v)=\frac{N(v)}{M(v)}
$$

where the numerator $N(v)$ is the noise inherent in the imaging process. The denominator $M(v)$ is the MTF of the synthesized optical instrument at the spatial frequency $v$. It is further known that both $N(v)$ and $M(v)$ are dependent on the trajectories of the constituent spacecraft of the MSIIS and that the noise term $N(v)$ is Gaussian. Some conditions on the noise process $e$ have obtained, specifically in terms of its second order moments, the mean squared error and the worst case error (WCE), such that some level of classification performance is guaranteed [5]. It is known that the functional dependence of $N(v)$ and $M(v)$ on the trajectories of the constituent spacecraft of an MSIIS is given by the following relationship $[3,4]$ :

$$
\begin{gathered}
M(v)=\sum_{m=1}^{n_{s}} \sum_{n=1}^{n_{s}} \int_{0}^{T} \hat{A}_{p}\left(v-\frac{\Delta r_{m n}(t)}{\lambda \bar{z}}\right) \mathrm{d} t \\
E|N(v)|^{2}=\sqrt{(}(1 / \mu) \sum_{m=1}^{n_{s}} \sum_{n=1}^{n_{s}} \int_{0}^{T}\left|\hat{A}_{p}\left(v-\frac{\Delta r_{m n}(t)}{\lambda \bar{z}}\right)\right|^{2} \mathrm{~d} t
\end{gathered}
$$

The following assumptions are made about the form of $\hat{A}_{p}$ and the frequency content of the image that is formed by the MSIIS.

Assumption II.1: The function $\hat{A}_{p}$ is assumed to have a value of unity within a circle of radius $\rho$ and zero elsewhere, that is,

$$
\hat{A}_{p}(x)= \begin{cases}1 & \text { if }\|x\| \leq \rho \\ 0 & \text { otherwise }\end{cases}
$$

Assumption II.2: The spatial frequency content of the images of interest is restricted to a circular disk of radius $R$ and $\rho \ll R$.

Let the frequency support of the images be denoted by $\Omega$. In light of the above assumptions, it can be seen that the following holds:

$$
E|e(v)|^{2}=\left\{1 / \mu \sum_{m} \sum_{n} \int_{0}^{T} \hat{A}_{p}\left[v-\frac{\Delta r_{m n}(t)}{\lambda \bar{z}}\right] \mathrm{d} t\right\}
$$

It was shown that to achieve a level of misclassification probability less than $p_{\min }$, it is sufficient that the WCE, $\|e\|_{\infty}$, obey the following inequality [3],

$$
\|e\|_{\infty} \leq C B^{2}
$$

where

$$
\|e\|_{\infty}=\left.\left.\left|\sup _{v \in \Omega}\right| e(v)\right|^{2}\right|^{1 / 2}
$$

$B$ is a constant depending on the feature to be identified and $C$ is a factor dependent on the probability of misclassification $p_{\min }$. Given a feature map for classification of the image, the probability of misclassification can be guaranteed to be less than $p_{\min }$ if [3]

$$
\left\{1 / \mu \sum_{m} \sum_{n} \int_{0}^{T} \hat{A}_{p}\left[v-\frac{\Delta r_{m n}(t)}{\lambda \bar{z}}\right] \mathrm{d} t\right\} \leq C B^{2}
$$

i.e.,

$$
\sum_{m} \sum_{n} \int_{0}^{T} \hat{A}_{p}\left[v-\frac{\Delta r_{m n}(t)}{\lambda \bar{z}}\right] \mathrm{d} t \geq \frac{1}{\mu C B^{2}}
$$

Denote $1 / \mu C B^{2}$ by $\bar{\Delta}$. From hereon in this paper, that the parameter $\bar{\Delta}$ is known shall be assumed. In Fig. 1, the relationship between the spacecraft positions is shown and the frequency coverage of the constellation in the spatial frequency plane is shown. Note that the constellation of spacecraft moving in the physical plane 

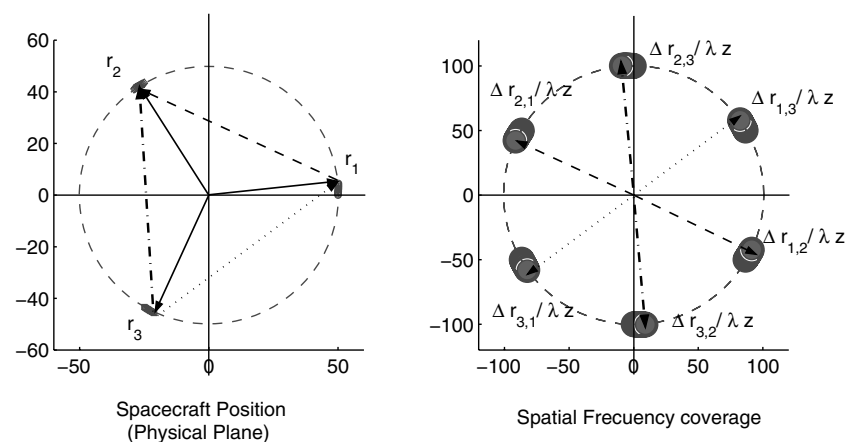

Fig. 1 Relationship between spacecraft positions and the spatial frequency coverage.

results in an associated constellation of coverage disks moving in the spatial frequency plane. The coverage disks are centered at the set of scaled relative distances corresponding to the constellation of spacecraft, that is, if the positions of the spacecraft are given by $\left\{r_{1}(t), \ldots, r_{n_{s}}(t)\right\}$, then the scaled spacecraft relative positions are given by $\left\{\left[\Delta r_{m n}(t)\right] / \lambda \bar{z}: \Delta r_{m n}(t)=r_{m}-r_{n}, m=1, \ldots, n_{s} ; n=\right.$ $\left.1, \ldots, n_{s}\right\}$ and a coverage disk (disk of radius $\rho$ ) is centered at each of these relative positions. The coverage disk located at the relative position $\left[\Delta r_{m n}(t)\right] / \lambda \bar{z}$ is denoted as the $(m, n)$ th coverage disk. The problem of designing a minimum-fuel maneuver for an MSIIS, such that the imaging constraints are satisfied can then be posed as follows:

Problem 1: Minimum-fuel maneuver for MSIIS (MF-MSIIS):

$$
\begin{gathered}
\min _{r_{1}, \ldots, r_{n_{s}}} \int_{0}^{T_{\max }} u^{T} u \text { subject to } \\
\sum_{m=1}^{n_{s}} \sum_{n=1}^{n_{s}} \int_{0}^{T} \hat{A}_{p}\left[v-\frac{\Delta r_{m n}(t)}{\lambda \bar{z}}\right] \mathrm{d} t \geq \bar{\Delta}, \quad \forall v \in \Omega \\
r_{i}(t)=\left[r_{i}^{1}(t), r_{i}^{2}(t)\right] ; r_{i}^{1}(t), r_{i}^{2}(t) \in L_{2}[0, T] \\
\dot{r}_{i}=f\left(r_{i}\right)+B u_{i}, u=\left[u_{1}, \ldots, u_{n_{s}}\right]^{T}
\end{gathered}
$$

Note that the above problem involves an uncountable number of integral constraints on the trajectories of the constituent spacecraft. In the following section, a painting problem is posed and its connection to the MF-MSIIS problem is examined.

\section{Image Formation as a Painting Problem: Relationship to the Traveling Salesman Problem}

In this section, the process of minimum-fuel image formation is posed as a painting problem. Specifically, it is shown that the process of image formation by an MSIIS is analogous to the painting of a large disk by smaller paintbrushes or "coverage disks." All the parameters defined in the previous section are unchanged in this section. Also, the following definitions hold:

Definition III.1: The "amount of paint" laid down by coverage disk $(m, n)$ at spatial frequency $v$ is defined as
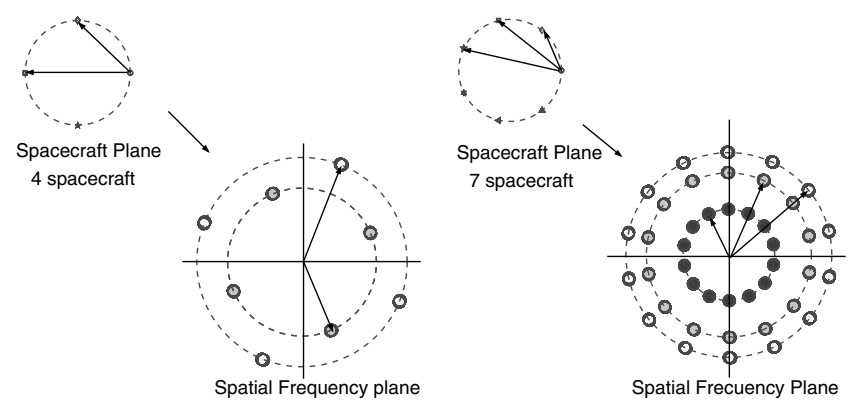

Fig. 2 Ring structure of the spatial frequency coverage of circular arrangement of spacecraft.

$$
\Delta_{m n}(v)=\int_{0}^{T} \hat{A}_{p}\left(v-\frac{\Delta r_{m n}}{\lambda \bar{z}}\right) \mathrm{d} t
$$

The following simplifying assumption is made:

Assumption III.1: The spacecraft are arranged symmetrically. The reasons for considering symmetric constellation are twofold. The first reason is that it simplifies the constellation design problem to that of designing the trajectories of a single spacecraft. The second reason is that though there is repeating coverage of the $u-v$ plane, it can be optimized such that only minimum amount of coverage is achieved at every frequency point.

In Fig. 2, the situation with four and seven spacecraft arranged symmetrically is shown. In the first case there are two rings of coverage disks, and the discs in the inner ring are twice covered. The inner ring is due to the relative trajectories of each spacecraft with respect to its adjacent spacecraft, and the outer ring is due to the relative trajectories of each spacecraft with respect to its opposite one. In the second case there are three rings and twice the number of discs per spacecraft in each. The fact that there is a multiple coverage in the $u-v$ plane, given the symmetry of the configuration it is taken into account by a constant $\beta$ that account for multiple coverage of the same point in the $u-v$ plane. The ratio of the radii of the two rings is fixed and determined by the geometry of the regular polygon along whose vertices the spacecraft are arranged. In the case of four spacecraft, it is $\sqrt{2}$. Similarly, for a higher number of spacecraft, there are a higher number of rings whose radii are in fixed ratios. Then, the amount of paint laid down by the various coverage disks can be partitioned as that being laid down by the different rings. It is shown for the case of four or five spacecraft, the problem is then easily generalized to higher number of spacecraft.

In the case of four or five spacecraft, there are two concentric rings. Denote the inner ring as ring I and the outer ring as ring II. Let $\Delta^{\mathrm{I}}$ and $\Delta^{\mathrm{II}}$ be the paint distribution laid down by ring I and ring II, respectively, that is, the total paint laid down by the coverage disks in the respective rings. The painting distributions $\Delta^{\mathrm{I}}$ and $\Delta^{\mathrm{II}}$ are not independent of each other. This can be seen from the constraint in the problem MF-MSIIS. Thus, $\Delta^{\mathrm{I}}=F\left(\Delta^{\mathrm{II}}\right)$, where $F$ is some fixed operator. Note that $\Delta^{\mathrm{I}}$ is a non-negative function and $F$ is a nonnegative operator. This operator $F$ depends on the number of spacecraft. The functional form of $F$ is found for four and five spacecraft MSIIS [3,4]. Any set of spacecraft trajectories lays down a particular paint distribution $\left(\Delta^{\mathrm{I}}, \Delta^{\mathrm{II}}\right)$. The trajectory constraints in MF-MSIIS are satisfied if the following "painting constraint" holds:

$$
\Delta^{\mathrm{I}}(v)+\Delta^{I I}(v) \geq \bar{\Delta}, \quad \forall v \in \Omega
$$

The central issue in the imaging problem is the coverage/painting constraint. As mentioned before, the imaging problem amounts to painting the resolution disk without leaving any gaps while maintaining a minimum thickness of paint. Thus, it follows that the paintbrushes or the coverage disks need to pass over every point in the resolution disk. This problem is kindred to the "covering salesman" or the coverage problem in robotic path planning $[16,17]$. In the aforementioned problem, the salesman or the agent must pass over all the points in the target environment. Note that in the case of imaging, the role of the salesman is played by the resolution disks or the paintbrushes while that of the target environment is played by the resolution disk. The coverage salesman problem is a variant of the "traveling salesman" problem and is known to be nondeterministic polynomial hard [16,17], that is, heuristics or approximate solutions have to be used while looking for the solution of these problems. Another perspective on this optimization problem can be obtained through the necessary conditions corresponding to the minimumfuel optimization problem 1 . The necessary conditions for the minimum-fuel problem can be formulated using Pontryagin's minimum principle and the Kuhn-Tucker theorem for optimization with inequality constraints. Problem 1 can be rewritten as follows: 


$$
\min _{u_{1}, \ldots, u_{n_{s}}} \int_{0}^{T_{\max }} u^{T} u \mathrm{~d} t, \text { subject to } \dot{\mathcal{X}}=f(\mathcal{X}, u), \quad q(T) \geq \bar{\Delta}
$$

where

$$
\mathcal{X}=\left[\begin{array}{l}
x \\
q
\end{array}\right]
$$

$x=\left[x_{1}, \ldots, x_{n_{s}}\right]^{T}$ is the state of the MSIIS, including the position and velocity of every spacecraft in the system, $q$ is defined as

$$
q=\left[q_{1}, \ldots, q_{K}\right]^{T} ; \quad \dot{q}_{k}=\mathcal{A}_{p}^{k}(x)=\sum_{n=1}^{n_{s}} \sum_{q=1}^{n_{s}} \hat{A}_{p}\left[v_{k}-\left(r_{m}-r_{n}\right)\right]
$$

and $u$ is a vector containing the control acting on each individual spacecraft.

The necessary conditions for the optimal solution of this problem are found to be

$$
\begin{gathered}
\dot{\lambda}_{1}=-\frac{\partial f^{T}}{\partial x} \lambda_{1}-\frac{\partial \mathcal{A}_{p}(x)^{T}}{\partial x} \lambda_{2} \\
\dot{\lambda}_{2}=0 \\
u=-\frac{1}{2} B^{T} \lambda_{1} \\
\dot{x}=f(x)+B u \\
\dot{q}_{k}=\mathcal{A}_{p}^{k}(x)
\end{gathered}
$$

where $\lambda_{1}$ is the vector of $6 n_{s}$ multipliers associated with $x$, and $\lambda_{2}$ is the vector of $K$ multipliers associated with $q$.

The boundary conditions of the problem are given as follows:

$$
\begin{aligned}
x(0) & =x_{o}, & q(0) & =0 \\
\lambda_{1}(T) & =0, & \lambda_{2}(T) & =-\mu
\end{aligned}
$$

where

$$
\mu_{k}=0, \quad \text { if }\left[\bar{\Delta}-q_{k}(T)\right]<0 ; \quad \mu_{k}>0, \quad \text { if }\left[\bar{\Delta}-q_{k}(T)\right]=0
$$

Recall that the variables $q_{k}$ correspond to the paint laid down at the frequency point $v_{k}$ and $\mu$ define the multipliers associated with these paint variables. As the number of these frequency points increases (note that there are an uncountable number of frequency points in the exact problem), the dimension of $\mu$ becomes very large. Thus, shooting methods for the solution of this problem are quite intractable because of the high dimensionality of the costate corresponding to the paint variables [18]. Hence, there is a need to obtain a solution that satisfies the paint constraints, that is, ensures that $q_{k}>\bar{\Delta}$ at the end of the maneuver. This corresponds to finding a trajectory that passes "close" to every one of the frequency points $v_{k}$, that is, the equivalent of finding a path that passes through all the cities in the traveling salesman problem. This is not a coincidence as the role of the cities in the traveling salesman problem is played by the frequency points in the optimal control formulation. Hence, there is a need to find classes of trajectories in the frequency plane that can satisfy the paint constraints.

Fortunately, the class of spiral trajectories, with a spiraling rate that is small enough, can satisfy this constraint quite trivially [i.e., $\left.q_{k}(T)>\bar{\Delta}\right]$. Hence, the design of minimum-fuel trajectories with respect to spiral maneuvers is considered in the next section and the corresponding optimization problem obtained, which is called the minimum-fuel pantograph.

\section{Minimum-Fuel Spiral Maneuvers}

In this section the problem of finding the minimum-fuel spiral maneuver that satisfies the imaging constraints is posed. For simplicity, two ring constellations are considered, that is, the case of four or five spacecraft. The treatment can easily be generalized to higher number of spacecraft. The spiral maneuvers are specified with respect to the outer ring (ring II).

Assumption IV.1: The spirals start close to the center and spiral out. Let $k(r)$ denote the rate at which ring II spirals out and let $\omega(r)$ denote the angular velocity of the spiral. Let $v_{r}$ and $v_{t}$ denote the radial and transverse velocity of the constellation, respectively. Note that the independent variable in this case can be the radius because it is monotonically increasing. Also, due to the symmetry of the configuration, the configuration of the constellation can be specified by $v_{r}$ and $v_{t}$ of any one of the spacecraft. Let the dynamics of the spacecraft be governed by the following dynamical equations:

$$
\begin{aligned}
& \dot{v}_{r}=f_{r}\left(r, v_{r}, v_{t}\right)+a_{r} \\
& \dot{v}_{t}=f_{t}\left(r, v_{r}, v_{t}\right)+a_{t}
\end{aligned}
$$

This is motivated by the free-space case, that is, perturbation free equations of motion. In the case of free-space note that $f_{r}=v_{t}^{2} / r$ and $f_{t}=-v_{t} v_{r} / r$. However, different dynamical regimes will require different dynamical formulations in general. Let the desired paint thickness be $\bar{\Delta}$. In the following, the painting/imaging constraints and the dynamical constraints in terms of the variables $v_{r}, v_{t}$ and $r$ are obtained. In the following it is assumed that the final radius of ring II, $R_{f}$, is given. Note that $R \leq R_{f} \leq \alpha R$, where $R$ is the radius of the resolution disk.

1) Painting/Imaging Constraints: Let the radius of the resolution disk be denoted by $R$. Let the ratio of the radii of the inner ring to the outer ring be denoted by $\alpha, \alpha>1$. Let the number of disks in the outer ring be denoted by $M$. Let the number of disks in the inner ring be denoted by $\beta M, \beta=1$ or $\beta=2$, depending on whether the number of spacecraft is odd or even. Let the thickness of paint laid down by the outer ring II at the point $(r, \theta)$ be denoted by $\Delta^{\mathrm{II}}(r, \theta)$. Similarly, let the paint laid down by inner ring I be denoted by $\Delta^{\mathrm{I}}(r, \theta)$. Let $A\left(r_{1}, r_{2}\right)$ represent the annular region between $r_{1}$ and $r_{2}$. Let $\rho$ denote the radius of the coverage disks, where it is assumed that $\rho \ll R$.

Definition IV.1: A paint distribution $\left(\Delta^{\mathrm{I}}, \Delta^{\mathrm{II}}\right)$ satisfies the fullpaint constraint (FPC) if

$$
\Delta^{\mathrm{II}}(r, \theta)+\Delta^{\mathrm{I}}(r, \theta) \geq \bar{\Delta}, \quad \forall(r, \theta) \in \Omega
$$

where $\bar{\Delta}$ represents the minimum desired thickness of the coat of paint and $\Omega$ represents the resolution disk.

Definition IV.2: The time spent by ring II in the annular region $A\left(r_{1}, r_{2}\right)$ is equal to the time spent by ring $\mathrm{I}$ in the region $A\left[\left(r_{1} / \alpha\right),\left(r_{2} / \alpha\right)\right]$.

Definition IV.3: It is assumed that the total volume of paint laid down by ring II in the region $A\left(r_{1}, r_{2}\right)$ is given by the expression $\pi \rho^{2} M T^{\mathrm{II}}\left(r_{1}, r_{2}\right) C$, where $T^{\mathrm{II}}\left(r_{1}, r_{2}\right)$ is the time taken by ring II to expand from $r_{1}$ to $r_{2}$. Similarly, the volume of paint laid down by ring $\mathrm{I}$ is given by $\pi \rho^{2} \beta M T^{\mathrm{I}}\left(r_{1}, r_{2}\right) C$.

Under the above assumptions, the painting constraints can be obtained as the following (details are given in previous literature $[4,5])$ :

$$
\begin{gathered}
\alpha \beta \frac{1}{v_{r}(r)}+\frac{1}{v_{r}\left(\frac{r}{\alpha}\right)} \geq \frac{r \bar{\Delta}}{\bar{C}}, \quad \forall r \in\left[0, R_{f}\right] \\
\frac{\mathrm{d} r}{\mathrm{~d} \theta}=\frac{r v_{r}(r)}{v_{t}(r)} \leq K
\end{gathered}
$$

Constraint on Eq. (24) is called the full painting constraint, and 
defines the requirement of a given amount of light collection on every region of $u-v$ plane coverage. Constraint on Eq. (25), defines a constraint in the maximum expansion rate of the spiral to prevent leaving gaps in the coverage of the $u-v$ plane.

2) Dynamical Constraints: If the independent variable is changed from time to the radius $r$, then the dynamical constraints can be written as follows:

$$
\begin{gathered}
v_{r}^{\prime}=\frac{f_{r}\left(r, v_{r}, v_{t}\right)}{v_{r}}+\bar{a}_{r} \\
v_{t}^{\prime}=\frac{f_{t}\left(r, v_{r}, v_{t}\right)}{v_{r}}+\bar{a}_{t}
\end{gathered}
$$

where $\bar{a}_{r}=\left(a_{r} / v_{r}\right)$ and $\bar{a}_{t}=\left(a_{t} / v_{r}\right)$ and all the derivatives have been changed from time derivatives to derivatives with respect to the radius.

3) Time Constraint: Assuming that a maximum time for the imaging maneuver $T_{\max }$ has been defined, the time constraint can be written as follows:

$$
\int_{0}^{R_{f}} \frac{\mathrm{d} r}{v_{r}(r)} \leq T_{\max }
$$

4) Fuel Usage: The fuel usage is given by the following expression,

$$
\int_{0}^{T_{\max }} \sqrt{a_{r}^{2}+a_{t}^{2}} \mathrm{~d} t
$$

Changing the independent variable to $r$ from $t$, the fuel usage for the constellation is obtained as follows:

$$
\int_{0}^{R_{f}} \sqrt{\bar{a}_{r}^{2}+\bar{a}_{t}^{2}} \mathrm{~d} r
$$

5) Minimum-Fuel Problem: With the above developments, the following proposition holds:

Proposition IV.1: Let assumptions IV.1-IV.3 hold. The solution of the generalized minimum-fuel panthograpic problem, for any number of spacecraft is given by the solution of the following problem:

$$
\min _{\bar{a}_{r}, \bar{a}_{t}} \int_{0}^{R_{f}} \sqrt{\bar{a}_{r}^{2}+\bar{a}_{t}^{2}} \mathrm{~d} r
$$

subject to

$$
\begin{gathered}
\alpha_{1} \beta_{1} \frac{1}{v_{r}\left(\frac{\alpha_{1} r}{\alpha_{n_{s}}}\right)}+\alpha_{2} \beta_{2} \frac{1}{v_{r}\left(\frac{\alpha_{2} r}{\alpha_{n_{s}}}\right)}+\ldots \geq \frac{r \bar{\Delta}}{\bar{C}}, \quad \forall r \in\left[0, R_{f}\right] \\
\frac{r v_{r}(r)}{v_{t}(r)} \leq K \\
v_{r}^{\prime}=\frac{f_{r}\left(r, v_{r}, v_{t}\right)}{v_{r}}+\bar{a}_{r} \\
v_{t}^{\prime}=\frac{f_{t}\left(r, v_{r}, v_{t}\right)}{v_{r}}+\bar{a}_{t} \\
\int_{0}^{R_{f}} \frac{\mathrm{d} r}{v_{r}(r)} \leq T_{\max }
\end{gathered}
$$

Thus, in this section the minimum-fuel problem for design of the trajectories of the constituent spacecraft of an MSIIS has been formulated. However, the optimization problem has bilinear constraints and thus is not a convex problem. However, if the spiraling rate, $k(r)=\mathrm{d} r / \mathrm{d} \theta$, of the spirals or the angular velocity $\omega(r)=\mathrm{d} \theta / \mathrm{d} t$ of the spiral, is fixed then the design of the optimal fuel trajectory can be shown to be a convex problem, under certain assumptions. This result is proved in the next section and is applied to the cases of deep space and linear dynamics.

\section{The Minimum-Fuel Pantograph}

As mentioned in the previous section, the minimum-fuel problem posed in the previous section contains bilinear constraints and as such is not a convex problem. In this section, the minimum-fuel optimization problems are posed, given that the spiraling rate $k(r)$ is fixed or the angular rate $\omega(r)$ is fixed. It shall be further shown that the two optimization problems so defined are convex, under certain assumptions on the dynamics of the system. First, the kinematic and the geometric design problems are defined, denoted as minimumfuel kinematic design (MFKD) and minimum-fuel geometric design (MFGD), respectively.

Definition V.1: The optimization problem obtained by fixing the spiraling rate $k(r)$, that is, the ratio $\left(r v_{r} / v_{t}\right)(r)$ in the minimum-fuel optimization problem (31) is defined to be the MFKD.

Definition V.2: The optimization problem obtained by fixing the angular rate $\omega(r)$, that is, the transverse velocity $v_{t}(r)$, in the minimum-fuel optimization problem, (31), is defined to be the MFGD.

The reason the problem is called a minimum-fuel pantograph is due to the pantographic imaging constraints in Eq. (32) [4,5]. This type of constraint relates a variable at a given position with its value at a different position. This type of constraint is equivalent to the timedelay constraint in the time domain. Note that either of the above optimization problems can be posed solely with respect to the radial velocity $v(r)$. In the following, two important classes of dynamics are considered, namely free space and dynamics of spacecraft in nearEarth orbits (i.e., the Clohessy-Wiltshire equations can be used to describe the relative position dynamics), and it is shown that the MFGD and MFKD are convex in both these cases.

Proposition V.1: First, consider the case of free space. The MFGD and MFKD are convex in free space.

Proof: The result is proved for the case of MFKD. The proof is very similar for the MFGD case.

$$
\begin{gathered}
f_{r}\left(r, v_{r}, v_{t}\right)=\frac{v_{t}^{2}}{r} \\
f_{t}\left(r, v_{r}, v_{t}\right)=-\frac{v_{r} v_{t}}{r}
\end{gathered}
$$

Let $\left[v_{r}(r) / v_{t}(r)\right]=k(r)$. Then $f_{r}\left\{r, v_{r},\left[k(r) / v_{r}(r)\right]\right\}=\left[v_{r} / r k^{2}(r)\right]$, which is linear and hence, convex in $v_{r}$. Similarly, $f_{t}\left\{r, v_{r},\left[k(r) / v_{r}\right]\right\}=\left[-v_{r} / r k(r)\right]$ is also linear in $v_{r}$ and hence, is convex in $v_{r}$. Thus, it follows that the MFKD is convex.

Now, consider the case of operation in near-Earth orbits. It is assumed that the plane of the circular orbit about which the perturbations of the spacecraft are considered is perpendicular to the target direction and thus, there is interest only in the in-plane motion of the satellites (this is a reasonable assumption for a target that is very far away from Earth). The motion of any satellite (due to symmetry, they will be staggered uniformly in phase) in the constellation can then be described in cylindrical coordinates (with respect to the circular orbit) [19] as follows:

$$
\begin{gathered}
\ddot{r}-2 n r_{0} \ddot{\theta}-3 n^{2} r+a_{r}=0 \\
r_{0} \ddot{\theta}+2 n \dot{r}+a_{t}=0 \\
\ddot{z}+n^{2} z=0
\end{gathered}
$$

where $r_{0}$ represents the radius of the circular orbit and $n$ represents the orbital mean motion [19]. Note that for the minimum-fuel problem as formulated in the previous section,

$$
v_{r}=\dot{r}
$$




$$
v_{t}=r \dot{\theta}
$$

However, the dynamical constraints of the minimum-fuel problem are reframed in terms of $v_{r}$ and $\omega=\dot{\theta}$ in order to ensure the convexity of the resulting minimum-fuel problem. In Eq. (39), changing the independent variable to $r$ instead of time and expressing the equations in terms of $v_{r}$ and $\omega$ results in the following equations for the in-plane motion of the satellites:

$$
\begin{gathered}
v_{r}^{\prime}-2 n r_{0} \frac{\omega}{v_{r}}-\frac{3 n^{2} r}{v_{r}}+\bar{a}_{r}=0 \\
r_{0} \omega^{\prime}+2 n+\bar{a}_{t}=0
\end{gathered}
$$

The out of plane motions of the spacecraft are not considered due to the reasons mentioned above. Now, consider the MFKD, that is, $(\mathrm{d} r / \mathrm{d} \theta)=$ const. Noting that $(\mathrm{d} r / \mathrm{d} \theta)=\left(v_{r} / \omega\right)$ it follows that the above equations are modified to

$$
\begin{gathered}
v_{r}^{\prime}-\frac{2 n r_{0}}{k(r)}-\frac{3 n^{2} r}{v_{r}}+\bar{a}_{r}=0 \\
r_{0}\left[\frac{k(r) v^{\prime}(r)-k^{\prime}(r) v(r)}{k^{2}(r)}\right]+2 n+\bar{a}_{t}=0
\end{gathered}
$$

Note that the above equations are both convex in $v_{r}$. Because all the other constraints are the same for the minimum-fuel problem as formulated in the previous section, it follows that the MFKD is convex for operation in near-Earth orbits. A similar result can also be proved for the MFGD. Thus, the above development can be presented as the following result:

Proposition V.2: The MFGD and MFKD are convex for nearEarth orbits, that is, if the governing equations of motion for the relative position dynamics of the satellite constellation are the Clohessy-Wiltshire equations.

Thus, in this section, it was shown that the conditions hold for the important cases of free-space and near-Earth operation in perturbed circular orbits. Though the case of two ring systems was considered, that is, the case of four or five spacecraft, the results are easily generalized to $n_{s}$ spacecraft. However, note that the general solution to the minimum-fuel problem is more difficult to obtain due to the bilinear nature of the pantographic constraints in terms of $v_{t}$ and $v_{r}$. A way to obtain a solution to the above problem is to solve a sequence of MFGDs and MFKDs recursively, that is, start with an initial value of the spiraling rate, $k_{0}(r)$, then design the optimal $v_{t}^{1}$ with respect to the given spiraling rate by solving an MFKD with respect to $k_{0}(r)$. On obtaining $v_{t}^{1}$, the optimal $k_{1}(r)$ can be obtained by solving the corresponding MFGD and so on to generate the spiraling and angular rates $\left[k_{n}(r), v_{t}^{n}(r)\right]$ until an equilibrium is reached. However, it is difficult to guarantee that the equilibrium is a minimum because there can be millions of such equilibria for optimization problems with bilinear constraints. However, many times, the interest is to obtain the best velocity profile with respect to a given spiraling rate, that is, solve an MFKD with respect to the given rate. The results so far show that such a problem can be conveniently solved, at least for the case of deep space and near-Earth operations, because it is a convex problem.

\section{Application: Fuel Optimal Spiral Trajectories in Deep Space}

In this section, the design of fuel optimal spiral trajectories in deep space is studied. Figure 3 shows the configuration of the type of symmetrical maneuver that was used for these examples. This algorithm can also be extended to nonsymmetrical configurations.

The dynamics of a satellite describing a spiral in deep space can be written in the form:

$$
\bar{a}_{r}(r)=\frac{a_{r}(r)}{v_{r}(r)}=\left(\frac{d v_{r}}{d r}-\frac{v_{t}}{k}\right)
$$
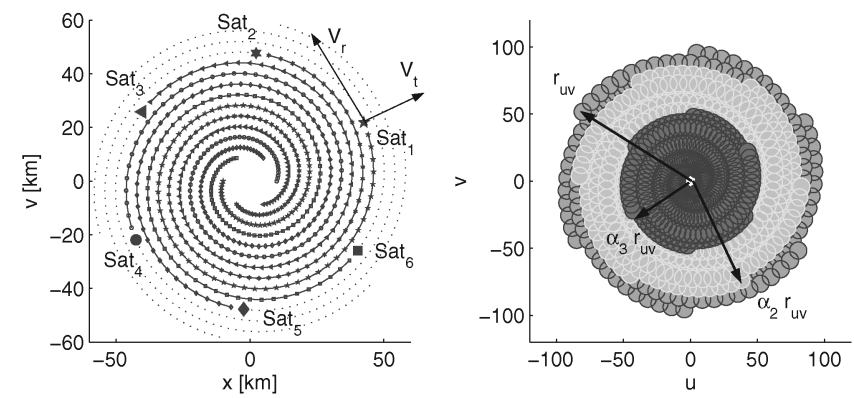

Fig. 3 Configuration of a spiral maneuver and its coverage in the $u-v$ plane. Notice the three coverage rings in this case.

$$
\bar{a}_{t}(r)=\frac{a_{t}(r)}{v_{r}(r)}=\left(\frac{d v_{t}}{d r}+\frac{v_{r}}{k}\right)
$$

To analyze the solutions to the fuel optimal problem, the optimization problem stated in Eqs. (31-36) is solved, for large times of maneuver (i.e., unconstrained or weakly constrained cases) and four satellites. The numerical solution was obtained for two cases. First, a suboptimal solution was obtained using $k$ constant, with $v_{r}$ as the optimization variable. In the second approach, an optimal solution was obtained with both $v_{r}$ and $v_{t}$ both as optimization variables.

Solutions were found for different values of the parameters: number of intervals, number of satellites and mission quality constraint. It was found in all the cases that the optimal solution corresponds to the loosest spiral, i.e., the spiral with the maximum possible spiraling rate.

Since the cost function is monotonically decreasing for any $k \geq 0$ it is expected that the value of $k$, which minimizes $J$, is attained at the constraint boundary; numerical solutions to the problem seem to corroborate these observations. Figure $4 \mathrm{a}$ and $4 \mathrm{~b}$ compare the solutions to the suboptimal problem with fixed radial rate (i.e., $k$ fixed) and the true optimal (i.e., $v_{r}$ and $v_{t}$ as optimization variables) problem. The solution on the left shows the velocities of a trajectory for a case where constraints are active, it clearly displays the discontinuity of the solution caused by the character of the constraint that acts only on part of the total radial distance. The plot on the right side shows the solution obtained for a case where the time of maneuver is higher and the constraints are not active. In both cases the value of $k$ lays at the constraint boundary. The small difference in the solution may be attributed to the discretization of the problem because in the optimal expansion rate case the solution converges to a value of $k$ for every $r$, that is close to the limit value up to the tolerance of the solving algorithm.

Hence, in accordance with the observations above, the following approximation is made:

Approximation 1: For a fixed time, the optimal fuel maneuver is obtained when $k$ is the maximum value allowed by the radial expansion limit, and the problem is thus reduced to the MFKD problem.

The dynamics of the system can then be written as

$$
\bar{a}_{r}(r)=\frac{a_{r}(r)}{v_{r}(r)}=\left(\frac{\mathrm{d} v_{r}}{\mathrm{~d} r}-\frac{v_{t}}{k}\right)
$$

$$
\bar{a}_{t}(r)=\frac{a_{t}(r)}{v_{r}(r)}=\left(\frac{r}{k} \frac{\mathrm{d} v_{r}}{\mathrm{~d} r}+2 \frac{v_{r}}{k}\right)
$$

and, for the cases under consideration, because the rate of change of velocity with respect to the radial distance is small, the contribution of $\mathrm{d} v_{r} / \mathrm{d} r$ can be assumed to be negligible. Figure 5 shows the small difference in the calculation of the acceleration when including the term. In the total cost function the contributions of these terms can be neglected and under the above approximations, Eq. (31) can be written as 

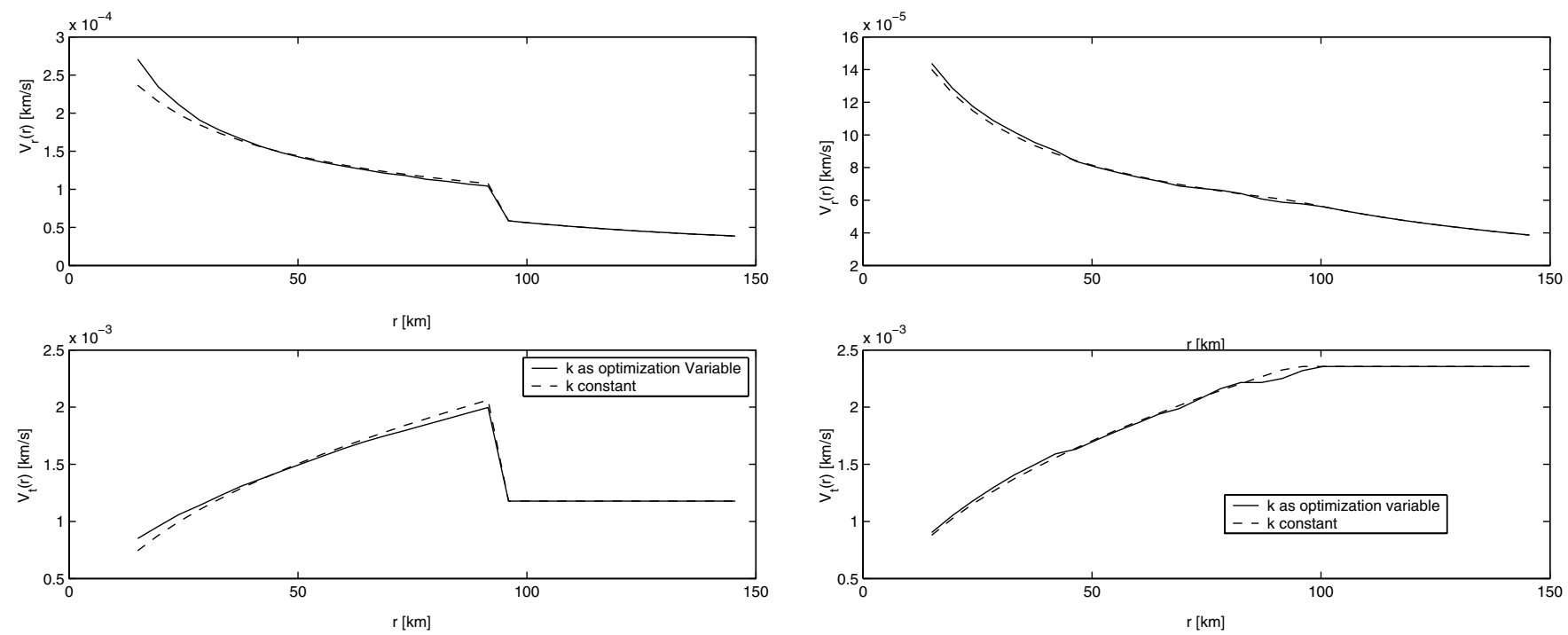

Fig. 4 Comparison of solutions for the fixed expansion rate and the optimal expansion rate cases for: a) Active constraints case (left), and b) Not active constraints (right).

$$
J=\int_{0}^{R_{\max }} \frac{v_{r}(r)}{k^{2}} \sqrt{r^{2}+4 k^{2}} \mathrm{~d} r
$$

\section{A. Indirect Method Solution for the Constrained Fuel Optimal Problem with $\boldsymbol{n}$ Spacecraft}

To obtain a solution to the minimum-fuel spiral maneuver problem, the problem is discretized as follows:

$$
\begin{gathered}
\Phi_{i}=\Phi\left(r_{i}\right), \quad i=1, \ldots, n_{s} \\
\Phi(r)=\frac{1}{v_{r}\left(\alpha_{m} r\right)}
\end{gathered}
$$

where $m$ is the number of rings in the configuration, namely $m=2$ for 4 and 5 spacecrafts, $m=3$ for 6 and 7 , and so on. The constants $\alpha_{i}$ (see Sec. IV) are proportionality constants for each ring, (e.g., $\alpha_{2}=\sqrt{2}$ for 4 spacecraft) and $\alpha_{M}$ is the value for the smallest ring in a given configuration. Following this discretization, the constraints can be written as linear inequality constraints Eqs. (57) and (63), as described below, and the numerical solution is formed using the sequential quadratic programming algorithm, fmincon and SNOPT in MATLAB. The constraints are

1) Full-Paint Constraint: The FPC, defined in the previous sections [refer to Eqs. (24) and (32)], can be written for the general case with $n$ spacecraft and $m$ rings as [6,7]:

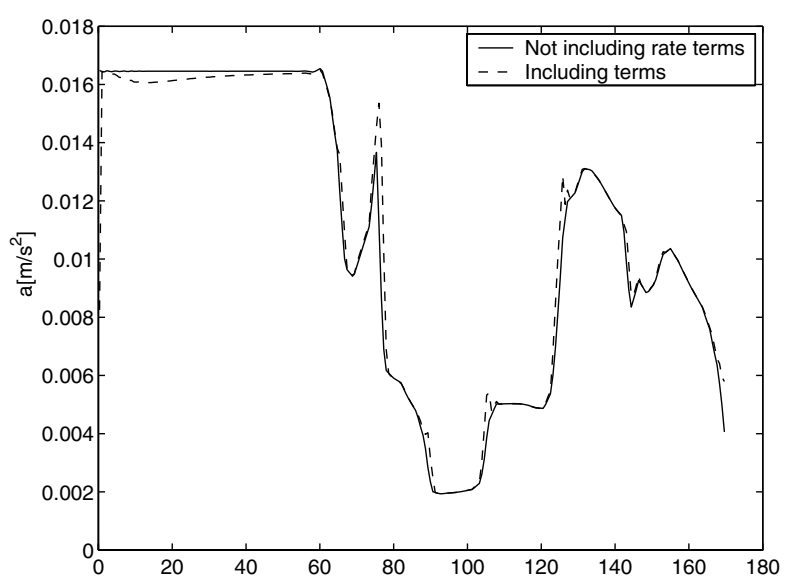

Fig. 5 Small influence of rate terms in total cost.

$$
\begin{aligned}
& G(r) K(r)+\alpha_{2} \beta_{2} G\left(\alpha_{2} r\right) K\left(\alpha_{2} r\right)+\ldots+\alpha_{m} \beta_{m} G\left(\alpha_{m} r\right) K\left(\alpha_{m} r\right) \\
& \quad \geq r \overline{\bar{\Delta}}
\end{aligned}
$$

where $\overline{\bar{\Delta}}$ is a constant proportional to the image quality. These constraints can be compacted into the following constraint:

$$
\begin{aligned}
& \Phi(r)+\alpha_{2} \beta_{2} \Phi\left(\alpha_{2} r\right)+\ldots+\alpha_{m} \beta_{m} \Phi\left(\alpha_{m} r\right) \\
& \quad \geq r \overline{\bar{\Delta}}, r \in\left(R_{\min }, R_{\max }\right)
\end{aligned}
$$

Thus, when the problem is discretized, the FPC reduces to the following linear constraint:

$$
\Gamma \cdot \underline{\Phi}(r) \geq \underline{r} \overline{\bar{\Delta}}
$$

where $\Gamma$ is a matrix given by

$$
\Gamma=\sum_{1}^{m} \alpha_{i} \beta_{i} \mathcal{W}_{\left(\alpha_{i} / \alpha_{m}\right)}
$$

Here $\alpha_{1}=1, \beta_{1}=1, m$ is the number of rings, and the matrix $\mathcal{W}_{\alpha}$ is the mapping in discrete space of the continuous function

$$
\mathcal{F}_{\alpha}[\Phi(r)]=\Phi(\alpha r)
$$

which has the form

$$
\Phi(\alpha r)=\mathcal{W}_{\alpha} \cdot \Phi(r)
$$

$$
=\left[\begin{array}{cccccccc}
1 & 0 & \ldots & 0 & 0 & \ldots & 0 & 0 \\
0 & \ldots & 0 & p & 1-p & 0 & \ldots & 0 \\
& & & \ldots & & & & \\
0 & \ldots & 0 & 0 & 0 & 0 & 0 & 1 \\
0 & 0 & 0 & 0 & 0 & 0 & 0 & 0 \\
& & & \ldots & & & & \\
0 & 0 & 0 & 0 & 0 & 0 & 0 & 0
\end{array}\right]\left[\begin{array}{c}
\Phi\left(r_{i}\right) \\
\Phi\left(r_{i+1}\right) \\
\cdot \\
\cdot \\
\cdot \\
\Phi\left(r_{n_{s}-1}\right) \\
\Phi\left(r_{n_{s}}\right)
\end{array}\right]
$$

where $p$ is a proportionality constant that calculates the value of $\Phi$ at the point $\alpha r$, as the linear interpolant of $\Phi\left(r_{i-1}\right)$ and $\Phi\left(r_{i}\right)$. As a particular case, $\mathcal{W}_{1}$ is the identity matrix.

2) Time Constraint: The time constraint is given by the integral in Eq. (36):

$$
\int_{0}^{R_{f}} \frac{\mathrm{d} r}{v_{r}(r)} \leq T_{\max }
$$


which after discretization, using a trapezoidal integration scheme, can be written as the vector product:

$$
V \cdot \Phi \leq T_{\max }
$$

where $V=\alpha_{M} \delta r\left[\begin{array}{llllll}0.5 & 1 & 1 & \ldots & 1 & 0.5\end{array}\right]$, and $\delta r$ is the distance between two contiguous discrete radial points.

\section{B. Results}

The minimum-fuel cost for MSIIS comprising 4 to 14 spacecraft, for different maximum times of maneuver, is displayed in Table 1, under the following imaging mission parameters:

1) $R=100$ kilometers

2) $\rho=0.02$

3) $C=2.5$

4) $B=2 \times 10^{-4}$

5) $\mu=1000$ photons $/ \mathrm{s}$

The imaging mission is to detect the bandedness of a Jupiter-sized planet at a distance of 25 light years. $50 \times 50$ pixel images of the planet are considered [5]. From the results, insight into the cost of MSIIS missions can be obtained, the order of magnitude of the required accelerations, and the general behavior of a maneuver covering the frequency plane. The results show that as the number of satellites is increased, a shorter time of maneuver is feasible, and the total fuel cost of an imaging maneuver is reduced. The maximum thrust required $\left(T_{\max }\right)$ is calculated per $100 \mathrm{~kg}$ mass of the constituent spacecraft and the fuel consumption is based on a specific impulse of $7000 \mathrm{~s}$ for an electrical propulsion system, that has been mentioned in some publications [20,21].

Some plots of the obtained solutions are shown in Figs. 6-9. The plots show the time history of the acceleration and the values of the corresponding velocities (refer to Fig. 3). The lower plot in these figures describes the fulfillment of the constraint, displaying the ratio of the time of light collection with respect to its requirement. The

Table 1 Cost for different number of satellite $\left(n_{s}\right)$ and different time of maneuver (time)

\begin{tabular}{|c|c|c|c|c|}
\hline$n_{s}$ & $\begin{array}{c}\text { Time } \\
\text { [hours] }\end{array}$ & $\Delta V[\mathrm{~m} / \mathrm{s}]$ & $\begin{array}{c}\mathrm{kg} \text { fuel } \\
(\mathrm{Isp}=7000 \mathrm{~s})\end{array}$ & $T_{\max }[\mathrm{mN}] / 100 \mathrm{~kg}$ \\
\hline 4 & 80 & 339 & 0.49 & 344 \\
\hline 4 & 60 & 947 & 1.38 & 438 \\
\hline 4 & 46 & 1337 & 1.94 & 2367 \\
\hline 5 & 80 & 104 & 0.15 & 3574 \\
\hline 6 & 60 & 207 & 0.303 & 487 \\
\hline 6 & 30 & 1138 & 1.62 & 2846 \\
\hline 7 & 80 & 27 & 0.039 & 19 \\
\hline 7 & 30 & 175 & 0.225 & 812 \\
\hline 8 & 80 & 209 & 0.304 & 69 \\
\hline 8 & 30 & 556 & 0.81 & 495 \\
\hline 8 & 24 & 696 & 1.01 & 774 \\
\hline 8 & 20 & 836 & 1.22 & 1128 \\
\hline 9 & 80 & 16 & 0.023 & 28 \\
\hline 9 & 30 & 122 & 0.178 & 406 \\
\hline 9 & 24 & 171 & 0.249 & 826 \\
\hline 10 & 80 & 135 & 0.198 & 45 \\
\hline 10 & 30 & 362 & 0.53 & 327 \\
\hline 10 & 24 & 510 & 0.744 & 453 \\
\hline 10 & 20 & 623 & 0.907 & 3009 \\
\hline 10 & 12 & 971 & 1.416 & 7463 \\
\hline 11 & 30 & 82 & 0.120 & 75 \\
\hline 11 & 24 & 104 & 0.152 & 139 \\
\hline 12 & 30 & 289 & 0.421 & 232 \\
\hline 12 & 24 & 361 & 0.527 & 363 \\
\hline 12 & 12 & 770 & 1.124 & 1749 \\
\hline 13 & 30 & 73 & 0.107 & 67 \\
\hline 13 & 24 & 91 & 0.134 & 104 \\
\hline 13 & 18 & 122 & 0.178 & 189 \\
\hline 13 & 12 & 212 & 0.310 & 2620 \\
\hline 14 & 30 & 209 & 0.306 & 203 \\
\hline 14 & 24 & 287 & 0.419 & 327 \\
\hline 14 & 12 & 576 & 0.839 & 1100 \\
\hline 14 & 8 & 862 & 1.258 & 2949 \\
\hline
\end{tabular}
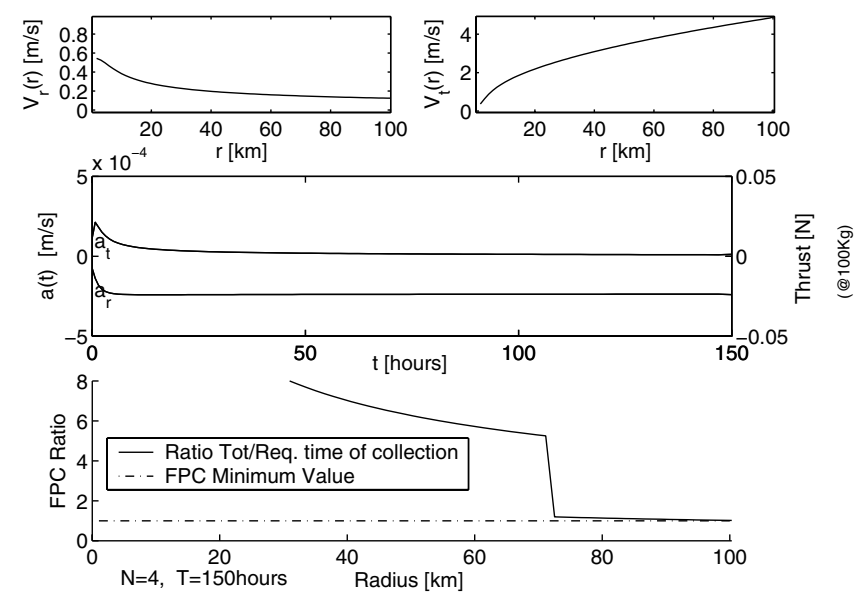

Fig. 6 State history for four spacecraft, $150 \mathrm{~h}$ FPC not active. Lower figure shows the fulfillment of FPC.
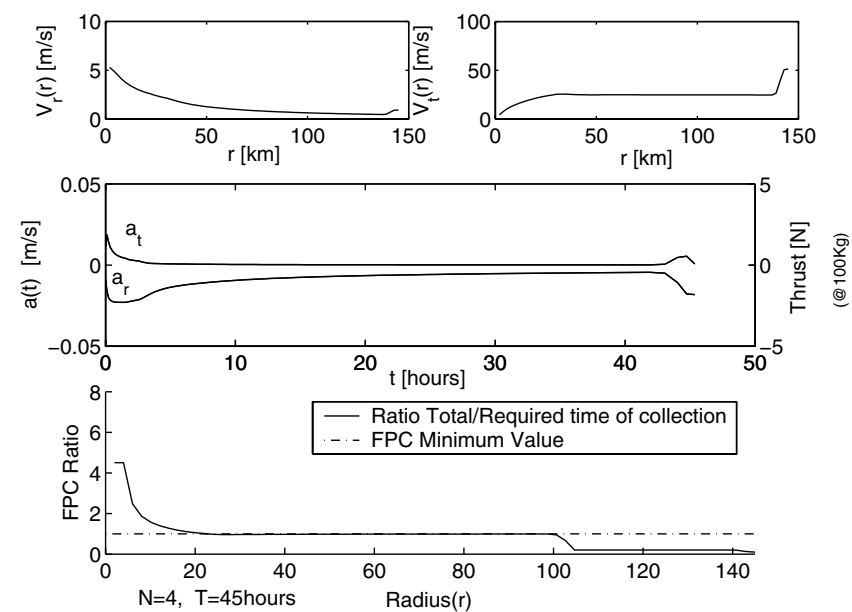

Fig. 7 State history for four spacecraft, $45 \mathrm{~h}$ maneuver with active constraints. Lower figure shows the fulfillment of FPC.
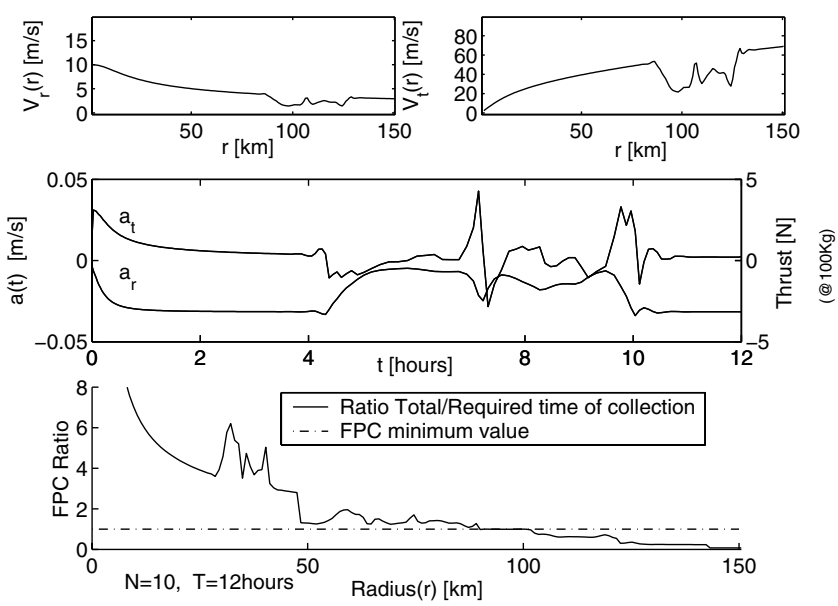

Fig. 8 State history for 10 spacecraft, $12 \mathrm{~h}$ maneuver with active constraints. Lower figure shows the fulfillment of FPC.

space trajectories of each of the maneuvers only depend on the value of $k$ (fixed for a given number of spacecraft). Figure 3 shows the maneuver for 6 spacecraft. In Fig. 6 the results for a nonconstrained case are shown, that is, a case in which the FPC is not active. The time of maneuver in this case is high. As the time of maneuver is reduced to the minimum feasible time, the optimal velocities increase and the FPC becomes active. Figure 7-9 show the solutions obtained for constrained low-time cases. In Fig. 7, a near-minimum time 

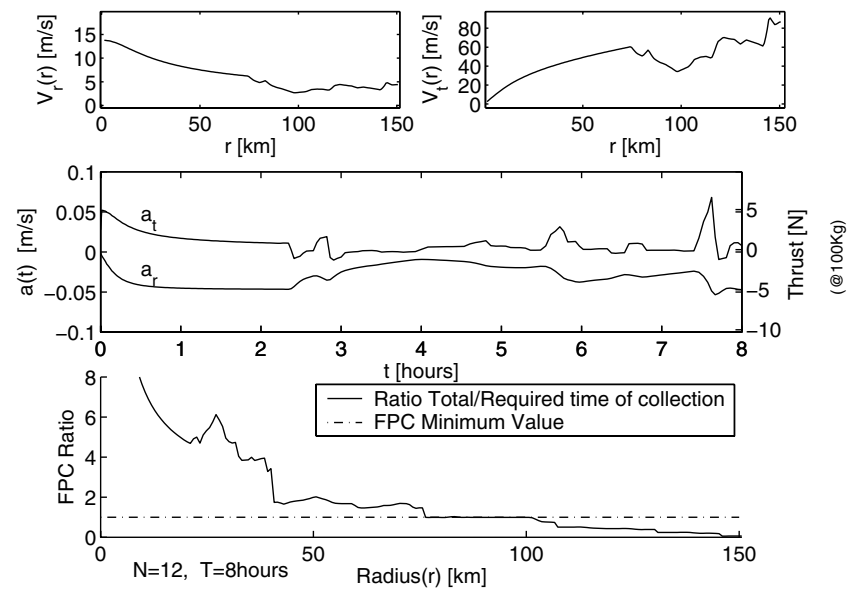

Fig. 9 State history for 12 spacecraft, $8 \mathrm{~h}$ maneuver with active constraints. Lower figure shows the fulfillment of FPC.

maneuver of $45 \mathrm{~h}$ is shown for a 10 spacecraft system, and in Figs. 8 and 9 , maneuvers are shown for 10 spacecraft in $12 \mathrm{~h}$ and for 12 satellites in $8 \mathrm{~h}$, respectively.

From the above mentioned results the actual cost of the imaging maneuver is calculated and the maximum acceleration required for such high resolution imaging missions. Maneuvers achievable in reasonable times are found to require reasonable fuel to total mass ratio. As an example, for a hundred of such maneuvers, using 8 spacecraft in $24 \mathrm{~h}$ missions, a spacecraft would require a ratio of fuel mass to total mass of

$$
\frac{M_{f}}{M_{t}}=1-e^{-\Delta V / I \cdot g}=0.63
$$

Figure 10 shows the results for the fuel to total mass ratio for 100 maneuvers, as a function of the number of spacecraft and for different times of maneuver. These estimated values are quite reasonable for such scientific missions because the particular imaging mission considered requires a very high level of light collection due to the feature selected as the criterion for classification [7]. Other missions would require much lower levels that would lead to less stringent time and fuel requirements. Maneuvers lasting $24 \mathrm{~h}$ comprising 13 spacecraft, require a maximum thrust of $100 \mathrm{mN}$ (per $100 \mathrm{~kg}$ of mass) and a total $\Delta V$ of the order of $0.09 \mathrm{~km} / \mathrm{s}$. Twenty-four hour maneuvers with fewer than nine satellites show a maximum required thrust of the order of $1 \mathrm{~N}$ (per $100 \mathrm{~kg}$ of total mass). Such high impulses are, however, practically infeasible using current electrical propulsion technologies due to the power supply constraints.

It was found that the accelerations required (and consequently, the required power), are reduced using more spacecraft in the maneuver and/or increasing the time of the maneuver. It is also found that the total cost of maneuver drops significantly when increasing the time of maneuver.

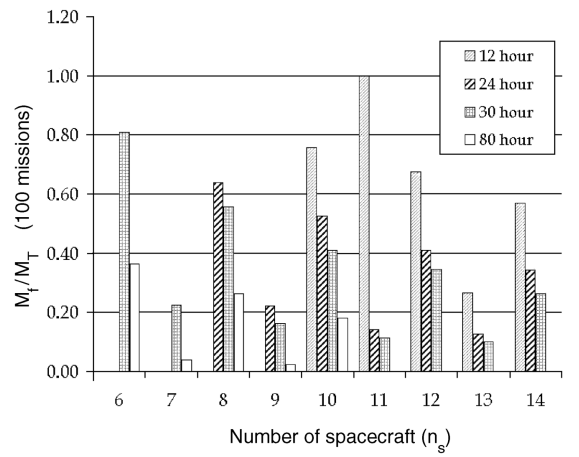

Fig. $10 M_{f} / M_{t}$ ratio for different number of spacecraft and different times of maneuver.
The fuel consumption for these maneuvers might be reduced with the development of more efficient electric propulsion systems with higher specific impulses and propulsion capacities like some that have been proposed for the exploration of outer solar planets [20,22]. Thus, the realization of such high resolution imaging systems would depend on a compromise between the number of spacecraft, time of maneuver, and the development of the appropriate propulsion technologies.

\section{Conclusions}

In this paper, it was shown that the minimum-fuel maneuver for a multispacecraft interferometric imaging system can be posed as a painting problem and it was argued that the optimization problem so obtained is computationally intractable because of its similarity to the traveling salesman problem and through an optimal control argument. Thus, in this paper, minimum-fuel spiral maneuvers were considered because spirals form a class of trajectories that satisfy the coverage constraints. The minimum-fuel optimization problem is nonconvex but it was shown that the kinematic design problem, obtained by fixing the spiraling rate in the minimum-fuel problem, and the geometric design problem, obtained by fixing the angular rate in the minimum-fuel problem, are both convex.

These results were applied to obtain fuel optimal solutions for MSIIS spiral maneuvers in deep space. The minimum-fuel spiral maneuvers for a specific deep space imaging mission was obtained. From the results, it can be concluded that such imaging maneuvers might be feasible provided that there is a compromise between the time of maneuver, the number of spacecraft, and the development of the current propulsion technologies.

A concern is the low level of acceleration that is required to maintain the minimum-fuel orbits. Thus, a high accuracy low impulse bit control is required. It is a concern that the order of the minimum accelerations is quite low, and current technologies under development providing low thrust might be important for the control of such maneuvers. A problem that should be addressed in future research is the study of the effect of inaccuracies in the imaging maneuver on the coverage of the frequency plane and consequently, the image quality. Also, the results need to be extended to other dynamical regimes such as near-Earth orbits and halo orbits.

\section{References}

[1] Quirrenbach, A., "Optical Interferometry," Annual Review of Astronomy and Astrophysics, Vol 39, Sept. 2001, pp 353-401.

[2] Borucki, W. J., Koch, D. G., Dunham, E. W., and Jenkins, J. M., "The Kepler Mission: A Mission to Determine the Frequency of Inner Planets near the Habitable Zone of a Wide Range of Stars," Planets Beyond Our Solar System and Next Generation Space Missions, edited by David Solderblom, Astronomical Society of the Pacific Conference Series, Baltimore, MD, , Vol. 119, 1997, pp. 153-173.

[3] Chakravorty, S., Kabamba P. T., and Hyland, D. C. "Guaranteed Classification Performance of Multi-Spacecraft Interferometric Imaging Systems," Journal of the Astronautical Sciences, Vol. 51, No. 2, 2003, pp. 205-226.

[4] Chakravorty, S., "Design and Optimal Control of Multi-Spacecraft Interferometric Imaging Systems," Ph.D. Thesis, Department of Aerospace Engineering, University of Michigan, Ann Arbor, MI, 2004.

[5] Chakravorty, S., Kabamba, P. T., and Hyland D. C., "Design of Minimum Time Maneuvers for Multi-Spacecraft Interferometric Imaging Systems," Proceedings of the Society of Photo-Optical Instrumentation Engineers, Modeling and Systems Engineering for Astronomy, Vol. 5497, No. 1, 2004, pp 427-436.

[6] Chakravorty, S., Kabamba, P. T., and Hyland, D. C., "Design of Minimum Time Maneuvers for Multi-Spacecraft Interferometric Imaging Systems," Journal of the Astronautical Sciences, Vol. 52, No. 3, 2004

[7] Chakravorty, S., Kabamba, P. T., and Hyland, D. C., "Modeling of Image Formation in Multi-Spacecraft Interferometric Imaging Systems," AIAA Space 2004 Conference and Exposition, Vol. 1, 2004, pp. 699-720; also AIAA Paper No. 2004-5895.

[8] Lawler, E. L., The Traveling Salesman Problem: A Guided Tour of Combinatorial Optimization, Wiley, New York, 1985. 
[9] Lien, Y., Ma, E., and Wah, B. W.-S., "Transformation of the Generalized traveling salesman Problem into the Standard traveling salesman Problem," Information Sciences, Vol. 74, 1993, pp. 177-189.

[10] Goodman, J. W., Introduction to Fourier Optics, McGraw-Hill, Boston, MA, 1996.

[11] Fitch, J. P., Synthetic Aperture Radar, Springer-Verlag, Berlin, 1987.

[12] Hussein, I. I., Scheeres, D., and Hyland, D. C., "Control of a Satellite Formation for Imaging Application," Proceedings of the American Control Conference, Vol. 1, 2003, pp. 308-313.

[13] Hussein, I. I., Scheeres, D., and Hyland, D. C., "Interferometric Observatories in Earth Orbit," Journal of Guidance, Control, and Dynamics, Vol. 27, No. 2, 2004, pp 297-301.

[14] Kong, E. M., Miller, D. W., and Sedwick, R. J., "Exploiting Orbital Dynamics for Aperture Synthesis Using Distributed Satellite Systems: Applications to Visible Earth Imager System," Journal of the Astronautical Sciences, Vol. 47, No. 1-2, 1999, pp. 53-75.

[15] DeCue, A. B., "Orbital Station-Keeping for Multiple Spacecraft Iinterferometry," Journal of the Astronautical Sciences, Vol. 39, No. 3,
1991, pp. 283-297.

[16] Arkin, E. M., and Hassin, R., "Approximation Algorithms for the Geometric Covering Salesman Problem," Discrete Applied Mathematics, Vol. 55, No. 3, 1994, pp. 197-218.

[17] Choset, H., "Coverage for Robotics: Survey of Recent Results," Annals of Mathematics and Artificial Intelligence, Vol. 31, 2001, pp. 113-126.

[18] Bryson, A. E., and Ho, Y. C., Applied Optimal Control, John Wiley \& Sons, New York, 1979.

[19] Wiesel, W. E., "Spaceflight Dynamics," 2nd ed., McGraw-Hill, New York, 1997.

[20] Patterson, M., "Herakles Thruster Development for the Prometheus JIMO Mission," AIAA Paper No. 2005-3890, 2005.

[21] Janosky, R., and Tverdokhlebov, S.,"High Power Hall Thrusters," NASA TM-1999-209436; also AIAA Paper No. 1999-2949 1999.

[22] Oleson, S., Ellion, F., Randolph, T., and Dipprey N., "The Electric Propulsion Segment of Prometheus 1," AIAA Paper No. 2005-3888, 2005. 